\title{
Therapy-Resistant Atypical Downbeat Nystagmus with Vertigo Confined to Specific Head-Hanging Positions: Mapping to the Gravity Vector on a Multi-Axis Turntable
}

\author{
Dominik Péus $^{\text {a, c }}$ Dominik Straumann ${ }^{d}$ Alexander Huber ${ }^{a}$ \\ Christopher J. Bockischa, d Vincent Wettstein ${ }^{a, e}$ \\ aDepartment of Otorhinolaryngology, Head and Neck Surgery, University of Zurich and \\ University Hospital Zurich, Zurich, Switzerland; 'b Department of Otorhinolaryngology, \\ Kantonsspital Baselland, Liestal, Switzerland; 'Department of Neurology, University of \\ Zurich and University Hospital Zurich, Zurich, Switzerland; dDepartment of Ophthalmology, \\ University of Zurich and University Hospital Zurich, Zurich, Switzerland; 'Rautipraxis AG, \\ Zurich, Switzerland
}

Keywords

Positional vertigo - Positional nystagmus - Downbeat nystagmus - Head-hanging position ·

Otoliths · Utriculus · Sacculus · Neurotology

\begin{abstract}
Downbeat nystagmus (DBN) observed in head-hanging positions, may be of central or peripheral origin. Central DBN in head-hanging positions is mostly due to a disorder of the vestibulo-cerebellum, whereas peripheral DBN is usually attributed to canalolithiasis of an anterior semicircular canal. Here, we describe an atypical case of a patient who, after head trauma, experienced severe and stereotypic vertigo attacks after being placed in various head-hanging positions. Vertigo lasted 10-15 s and was always associated with a robust DBN. The provocation of transient vertigo and DBN, which both showed no decrease upon repetition of maneuvers, depended on the yaw orientation relative to the trunk and the angle of backward pitch. On a motorized, multi-axis turntable, we identified the two-dimensional Helmholtz coordinates of head positions at which vertigo and DBN occurred ( $y$-axis: horizontal, spacefixed; $z$-axis: vertical, and head-fixed; $x$-axis: torsional, head-fixed, and unchanged). This twodimensional area of DBN-associated head positions did not change when whole-body rotations took different paths (e.g., by forwarding pitch) or were executed with different velocities. Moreover, the intensity of DBN was also independent of whole-body rotation paths and velocities. So far, therapeutic approaches with repeated liberation maneuvers and cranial vibrations were not successful. We speculate that vertigo and DBN in this patient are due
\end{abstract}


to macular damage, possibly an unstable otolithic membrane that, in specific orientations relative to gravity, slips into a position causing paroxysmal stimulation or inhibition of macular hair cells.

\section{Introduction}

Downbeat nystagmus (DBN) is the most common central spontaneous nystagmus. During clinical examination, cerebellar DBN can be modulated by changing the patient's head position relative to gravity. The upward ocular drift in cerebellar DBN consists of 2 components: one is an eye position-independent upward velocity bias, the other is a vertical gaze-evoked drift due to an impaired vertical neural integrator [1]. In most patients, the DBN is most pronounced in a prone and side position and least intense in a supine position [2]. Central DBN, in contrast to peripheral DBN, does not fade away after positional maneuvers and is mostly associated with less subjective vertigo $[3,4]$. Peripheral DBN is usually a positioning nystagmus and associated with rotational vertigo [5]. Possible labyrinthine pathomechanisms of positioning DBN are canalolithiasis of the anterior semicircular canal and apogeotropic posterior canal benign paroxysmal positional vertigo (BPPV) $[6,7]$. Here, we describe an atypical case of a patient who, after head trauma, experienced severe and stereotypic vertigo attacks after being placed in various head-hanging positions, which cannot be explained by the mechanisms of canalithiasis.

\section{Case Presentation}

\section{Patient History}

A 41-year-old man was assigned in our department with a 2 months history of stereotypic vertigo attacks. The symptoms appeared immediately after a head concussion. The initial CT scan of the head at the emergency department was without pathological findings. Due to persistent symptoms, a cranial MRI scan of the head was performed, also with normal findings. The attacks were triggered by a specific change in head position, namely when lying down into a supine position, or when looking upwards by reclining his head in sitting or standing position. He reported having a spinning sensation and a severely blurred vision during the attacks and an increasing feeling of nausea with repeated attacks. The patient was otherwise in full health and worked in a bakery. Due to vertigo, his general practitioner put him on permanent sick leave after the accident.

\section{Clinical Examination and Vestibular Findings}

Apart from positioning maneuvers, the clinical neurotological, extended neurological, and ENT examination showed normal findings throughout. The video head impulse tests for all 6 semicircular canals showed normal gain values ( $>0.8$ for horizontal and $>0.7$ for LARP and RALP planes) and no suspicious catch-up saccade patterns. The dynamic visual acuity test was also normal to both sides. Cervical vestibular evoked myogenic potentials showed a normal, symmetric reflex threshold of $90 \mathrm{~dB}$ SPL bilaterally. Ocular cervical vestibular evoked myogenic potential showed normal reflexes bilaterally with a $27 \%$ asymmetry ratio to the left side, which was interpreted as normal (cutoff 35\%). Normal cyclorotation of the eyes was found in fundus photography. Video nystagmography showed normal ocular motion in 
smooth pursuit and saccade testing, absent head-shaking nystagmus, and a strong reaction to caloric ear irrigation on both sides without pathological asymmetry. The testing of the subjective visual vertical showed a slight tilt $\left(2.2^{\circ}\right)$ toward the left side (normal range $<1.9^{\circ}$ ). Pure tone audiometry was normal and did not suggest any damage due to contusion labyrinth.

The Hallpike maneuver to the right side and in the sagittal plane triggered a violent down-beating nystagmus without torsional component after a latency of about $2 \mathrm{~s}$, which decreased and faded out after about $15 \mathrm{~s}$. At left and right gaze during the attack, the nystagmus remained purely vertical in an eye-fixed reference frame, with no additional torsional component. This pattern fits the geometric requirements for the (otolith mediated) vertical translational VOR. Interestingly, the velocity of the maneuver did not affect the intensity of the nystagmus. Even when reclining head and body very slowly, the strong nystagmus occurred after reaching about $100^{\circ}$ head reclination $\left(0^{\circ}\right.$ : upright; $90^{\circ}$ : supine $)$ sitting up from the head-hanging position did not trigger an upbeat nystagmus, independent the velocity of the procedure. To determine, whether pressure or circulation of ICF and blood was of influence, a slow head reclination to the left Hallpike position was performed, which triggered no nystagmus. Then the patient's legs were then lifted up (Trendelenburg's maneuver) to increase venous pressure. After a few seconds, the described nystagmus occurred.

\section{Attempted Treatments}

First, we suspected an anterior canal BPPV, which is often described with predominantly down-beating nystagmus in the literature [6]. Accordingly, we performed multiple liberation procedures for the anterior canals such as the Yakovino maneuver [8] and $360^{\circ}$ forward somersault maneuvers, as well as classical and extended maneuvers for all canals in several multi-axis turntable sessions. Overall, neither the quality of the nystagmus nor the positions in which the nystagmus was triggered could be altered. With these findings, a pressure-triggered, position-dependent cause was suspected, such as a possible third window of the labyrinth. However, other signs such as Tulio phenomenon or pressure-induced nystagmus, autophony, and hyperacusis were lacking. A new high-resolution CT of the temporal bone revealed a bony dehiscence of the right posterior SCC. Computed reconstruction in the corresponding plane showed that in supine and right Hallpike position, the dehiscence came to place at the lowest point of the SCC, possibly rendering it most susceptible to alteration of pressure and blood circulation. A symptomatic posterior semicircular canal dehiscence was postulated, and after thorough evaluation, the patient wished to take the chance of a surgical occlusion. A plugging and covering of the dehiscence in a transmastoidal, posterior fossa approach was then performed without complications and full hearing preservation. Postoperative video head impulse test showed an isolated right posterior SCC function loss as a sign of successful occlusion. Unfortunately, the described symptoms and clinical findings persisted. A new MRI again confirmed normal neurocranial findings.

\section{Mapping to the Gravity Vector}

To better understand the possible origin of the patient's symptoms, we conducted a positional mapping on the motorized multi-axis turntable, which allowed whole-body rotations relative to gravity (see online suppl. Video, Supplemental Digital Content; for all online suppl. material, see www.karger.com/doi/10.1159/000517840, which shows an example measurement). The aim was to locate the exact positions and paths, by which the nystagmus and vertigo were triggered. Eye movements were recorded by video nystagmography (EyeSeeCam, Interacoustics). The center of the head was positioned at the center of the turntable's rotation axes. Two-dimensional Helmholtz coordinates of head positions at which

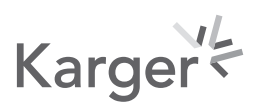


Fig. 1. A motorized multi-axis turntable was used for the gravity vector mapping. We used two-dimensional Helmholtz coordinates of head positions to summarize where vertigo and DBN occurred ( $y$-axis: horizontal, space-fixed; $z$-axis: vertical, head-fixed; $x$-axis: torsional, head-fixed, and unchanged). The dots stand for the onset of nystagmus in each positioning maneuver. The red dots refer to a backward pitch with the head-yaw axis fixed. The yellow dots refer to the head roll toward the midline, lying $120^{\circ}$ supine, and space fixed. The blue dot refers to the forward rotation along with the pitch, left ear anterior, and right ear posterior.

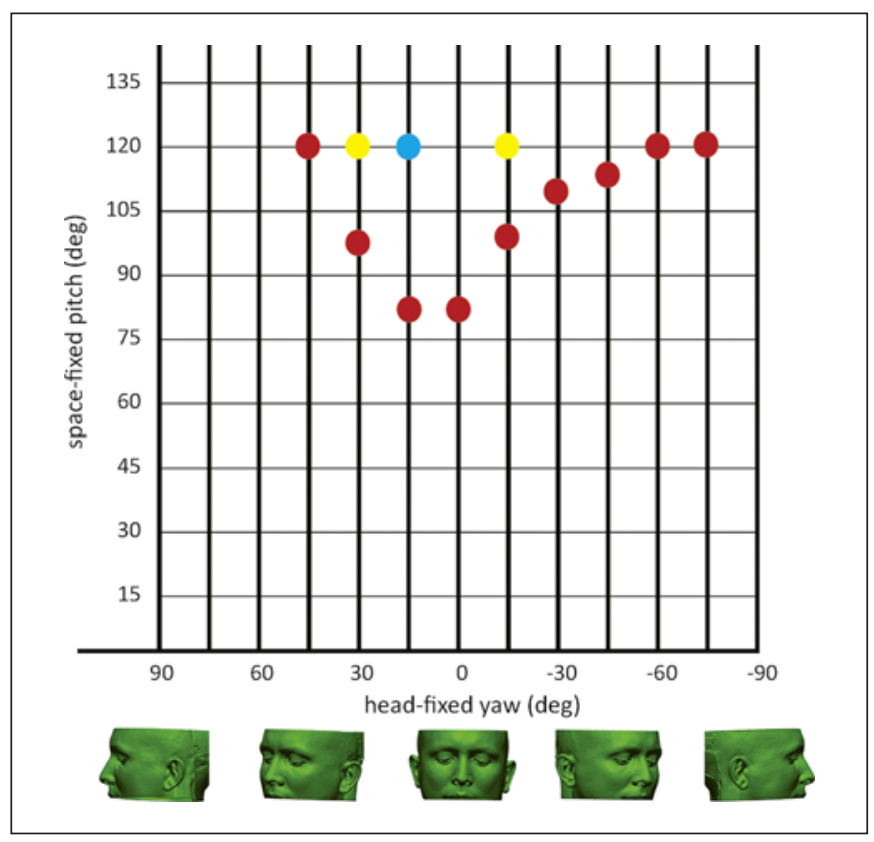

vertigo and DBN occurred were identified (y-axis: horizontal, space-fixed; $z$-axis: vertical, head-fixed; $x$-axis: torsional, head-fixed, unchanged). This two-dimensional area of DBNassociated head positions did not change when whole-body rotations took different paths (e.g., by forwarding pitch) or were executed with different velocities. The mapping is summarized in Figure 1. Approaching this area from any direction and with any velocity triggered the same nystagmus, that is, forward and backward in the tilt axis, as well as from left and right in the head-yaw axis. The quality, direction, and intensity of the nystagmus and vertigo could not be altered by any parameter. When triggered, the same nystagmus occurred every time.

\section{Discussion}

The mapping analyzed the onset of nystagmus and vertigo as a function of head position relative to gravity. It revealed a well-defined trigger area described by horizontal and vertical Helmholtz coordinates. The sensation of vertigo lasted $10-15 \mathrm{~s}$ and was associated with a robust DBN. The provocation of transient vertigo and DBN, both of which showed no decrease upon repetition of maneuvers, depended on the yaw orientation relative to the trunk and the angle of backward pitch. While central DBN typically persists when the patient's head remains in the hanging position, which is termed as positional nystagmus, peripheral DBN lasts for a few seconds only, which is termed as positioning nystagmus. In our case, some features such as the relative symmetry of the trigger area, the absence of a torsional component of the nystagmus, and the lack of an existing, corresponding model of canalithiasis pathomechanism tempted to consider a central cause. On the other hand, other features such as the rapid onset and short duration of nystagmus, which is very unusual for a central cause of DPN, as well as uniform consistency over the years in the absence of any central sign and with normal cranial MRI findings, argue against a central cause. Also, the fact that the DBN is only triggered in supine position argues for a peripheral origin because central DBN is more pronounced in prone and side positions and least in a 
supine position [2].Studies investigating the natural course of positioning DBN of noncentral, that is, idiopathic cause showed varying or additional findings over time, such typical posterior canal BPPV $[3,5]$, which was not the case in our patient. Also, spontaneous resolution over time was shown in almost all patients [5].

Based on our findings, we postulate a labyrinthine origin, but given the described detailed characteristics of this particular case, we were not able to attribute any existing diagnosis to our patient. We postulate a damage to the utricular or saccular macula, such as an unstable otolithic membrane that, when entering a confined trigger area relative to the gravitational axis, slips or collapses into a position causing blunt stimulation of macular hair cells, ultimately causing paroxysmal DBN and vertigo. We support this hypothesis by studies that showed vertical and horizontal nystagmus upon isolated saccular or utricular stimulation [9-12]. Since it is commonly accepted that the sacculus is merely responsible for detecting linear motion in the vertical plane, our hypothesis tends toward a pathology of the saccular macula. The described pathomechanism could explain why even slow positioning into the trigger area leads to strong nystagmus. It could also explain the fact that symptoms did not resolve over time. Since there is no possibility to investigate the macular integrity in vivo in humans, we cannot confirm our hypothesis with today's standards. Moreover, the cause of the possible lesion stays unclear.

\section{Conclusion}

To our knowledge, this is the first and only case of a patient with MRI-negative, stereotypic paroxysmal positioning DBN. We were challenged by a persistent disease that did not improve on any of our treatments or over time. Our goal was, therefore, to investigate this case as thoroughly as possible in order to understand the pathophysiological mechanism of these novel findings. With future diagnostic possibilities, we hope to gain more insight into such complex cases, and we encourage researchers with similar cases to report on their findings and conclusions, in order to find possible treatment solutions for the future.

\section{Statement of Ethics}

The study was conducted according to the criteria by the declaration of Helsinki. Written informed consent was obtained from the patient for publication of this case report, any accompanying images, and also for any supplementary video materials. The study is exempt from Ethical Committee approval because the investigations did not differ from standard medical care.

\section{Conflict of Interest Statement}

The authors have no conflicts of interest to declare.

\section{Funding Sources}

This research did not receive any specific grant from funding agencies in the public, commercial, or not-for-profit sectors.

\section{Karger'}




\section{Author Contributions}

D.P., D.S., and V.W. oversaw the patient and collection of data. D.P and D.S. created the figures. A.H. performed the surgery. D.P., D.S., A.H., and V.W. wrote and critically revised the manuscript.

\section{Availability of Data and Material}

All data generated or analyzed during this study are included in this article and its supplementary material files. Further enquiries can be directed to the corresponding author.

\section{References}

1 Glasauer S, Dieterich M, Brandt T. Central positional nystagmus simulated by a mathematical ocular motor model of otolith-dependent modification of Listing's plane. J Neurophysiol. 2001;86(4):1546-54.

2 Marti S, Palla A, Straumann D. Gravity dependence of ocular drift in patients with cerebellar downbeat nystagmus. Ann Neurol. 2002;52(6):712-21.

3 Bertholon P, Bronstein AM, Davies RA, Rudge P, Thilo KV. Positional down beating nystagmus in 50 patients: cerebellar disorders and possible anterior semicircular canalithiasis. J Neurol Neurosurg Psychiatry. 2002; 72(3):366-72.

4 Tarnutzer AA, Straumann D. Nystagmus. Curr Opin Neurol. 2018;31(1):74-80.

5 Cambi J, Astore S, Mandalà M, Trabalzini F, Nuti D. Natural course of positional down-beating nystagmus of peripheral origin. J Neurol. 2013;260(6):1489-96.

6 Califano L, Salafia F, Mazzone S, Melillo MG, Califano M. Anterior canal BPPV and apogeotropic posterior canal BPPV: two rare forms of vertical canalolithiasis. Acta Otorhinolaryngol Ital. 2014;34(3):189-97.

7 Vannucchi P, Pecci R, Giannoni B, Di Giustino F, Santimone R, Mengucci A. Apogeotropic posterior semicircular canal benign paroxysmal positional vertigo: some clinical and therapeutic considerations. Audiol Res. 2015; 5(1):130.

8 Yacovino DA, Hain TC, Gualtieri F. New therapeutic maneuver for anterior canal benign paroxysmal positional vertigo. J Neurol. 2009;256(11):1851-5.

9 Goto F, Meng H, Bai R, Sato H, Imagawa M, Sasaki M, et al. Eye movements evoked by selective saccular nerve stimulation in cats. Auris Nasus Larynx. 2004;31(3):220-5.

10 Goto F, Meng H, Bai R, Sato H, Imagawa M, Sasaki M, et al. Eye movements evoked by the selective stimulation of the utricular nerve in cats. Auris Nasus Larynx. 2003;30(4):341-8.

11 Hitier M, Sato G, Zhang YF, Zheng Y, Besnard S, Smith PF. Vestibular-related eye movements in the rat following selective electrical stimulation of the vestibular sensors. J Comp Physiol A Neuroethol Sens Neural Behav Physiol. 2018;204(9-10):835-47.

12 Hageman KN, Chow MR, Roberts D, Boutros PJ, Tooker A, Lee K, et al. Binocular 3D otolith-ocular reflexes: responses of chinchillas to prosthetic electrical stimulation targeting the utricle and saccule. J Neurophysiol. 2020;123(1):259-76.

\section{Karger' ${ }^{\prime}$}

